\title{
On a subclass of starlike functions associated with a vertical strip domain
}

\author{
Yong Sun ${ }^{1}$, Zhi-Gang Wang ${ }^{2 *}$ (D) Antti Rasila ${ }^{3}$ and Janusz Sokół ${ }^{4}$
}

"Correspondence:

zhigangwang@foxmail.com

${ }^{2}$ School of Mathematics and Computing Science, Hunan First

Normal University, Changsha, People's Republic of China

Full list of author information is

available at the end of the article

\begin{abstract}
In this paper, we consider a subclass of starlike functions associated with a vertical strip domain. We obtain several results concerned with integral representations, convolutions, and coefficient inequalities for functions belonging to this class. Furthermore, we consider radius problems and inclusion relations involving certain classes of strongly starlike functions, parabolic starlike functions, and other types of starlike functions. The results are essential improvements of the corresponding results obtained by Kargar et al., and the derivations are similar to those used earlier by Sun et al. and Kwon et al.
\end{abstract}

MSC: Primary 30C45; secondary 30C55

Keywords: Analytic function; Univalent function; Starlike function; Differential subordination

\section{Introduction}

Let $\mathcal{A}$ denote the class of the functions of the form

$$
f(z)=z+\sum_{n=2}^{\infty} a_{n} z^{n}
$$

which are analytic and univalent in the open unit disk $\mathbb{U}=\{z \in \mathbb{C}:|z|<1\}$. A function $f \in \mathcal{A}$ is said to be starlike of order $\beta(0 \leq \beta<1)$ if it satisfies the condition

$$
\Re\left(\frac{z f^{\prime}(z)}{f(z)}\right)>\beta \quad(z \in \mathbb{U}) .
$$

We denote by $\mathcal{S}^{*}(\beta)$ the class of starlike functions of order $\beta$. A function $f \in \mathcal{A}$ is said to be convex of order $\beta(0 \leq \beta<1)$ if it satisfies the condition

$$
\Re\left(1+\frac{z f^{\prime \prime}(z)}{f^{\prime}(z)}\right)>\beta \quad(z \in \mathbb{U}) .
$$

We denote by $\mathcal{K}(\beta)$ the class of convex functions of order $\beta$. For simplicity, we also use the notations $\mathcal{S}^{*}:=\mathcal{S}^{*}(0)$ and $\mathcal{K}:=\mathcal{K}(0)$.

A function $f \in \mathcal{A}$ is said to be strongly starlike of order $\gamma(0 \leq \gamma<1)$ if

$$
\left|\arg \left(\frac{z f^{\prime}(z)}{f(z)}\right)\right| \leq \frac{\pi}{2} \gamma \quad(z \in \mathbb{U}) .
$$

(c) The Author(s) 2019. This article is distributed under the terms of the Creative Commons Attribution 4.0 International License (http://creativecommons.org/licenses/by/4.0/), which permits unrestricted use, distribution, and reproduction in any medium, provided you give appropriate credit to the original author(s) and the source, provide a link to the Creative Commons license, and indicate if changes were made. 
We denote by $\mathcal{S} \mathcal{S}(\gamma)$ the class of strongly starlike functions of order $\gamma$. We also consider the subclass $\mathcal{P S} \subset \mathcal{A}$ of parabolic starlike functions in $\mathbb{U}$ (see [8]), which satisfy the inequality

$$
\left|\frac{z f^{\prime}(z)}{f(z)}-1\right| \leq \Re\left(\frac{z f^{\prime}(z)}{f(z)}\right) \quad(z \in \mathbb{U}) .
$$

Recall that an analytic function $w$ in the unit disk $\mathbb{U}$ is a Schwarz function if it satisfies the conditions of the Schwarz lemma:

$$
w(0)=0 \quad \text { and } \quad|w(z)|<1 \quad(z \in \mathbb{U})
$$

For two analytic functions $f$ and $g$ in $\mathbb{U}$, we say that the function $f$ is subordinate to $g$ in $\mathbb{U}$ and write

$$
f(z) \prec g(z) \quad(z \in \mathbb{U})
$$

if there exists a Schwarz function $w(z)$ such that

$$
f(z)=g(w(z)) \quad(z \in \mathbb{U})
$$

It is well known that if $f(z) \prec g(z)(z \in \mathbb{U})$, then $f(0)=g(0)$ and $f(\mathbb{U}) \subset g(\mathbb{U})$. Furthermore, if the function $g$ is univalent in $\mathbb{U}$, then we have the equivalence

$$
f(z) \prec g(z) \quad(z \in \mathbb{U}) \quad \Longleftrightarrow \quad f(0)=g(0) \quad \text { and } \quad f(\mathbb{U}) \subset g(\mathbb{U}) \text {. }
$$

In 1998, Sokół [11] introduced the class $\mathcal{S} \mathcal{L} \subset \mathcal{S}^{*}$ consisting of the functions $f \in \mathcal{A}$ such that

$$
\frac{z f^{\prime}(z)}{f(z)} \prec \sqrt{1+z} \quad(z \in \mathbb{U})
$$

Recently, Kargar et al. [2] investigated the class $\mathcal{M S}(\alpha)$ (see Definition 1) and obtained several radius results for certain well-known function classes.

Definition 1 A function $f \in \mathcal{A}$ is said to belong to the class $\mathcal{M S}(\alpha)(\pi / 2 \leq \alpha<\pi)$ if it satisfies the following conditions:

$$
1+\frac{\alpha-\pi}{2 \sin \alpha}<\Re\left(\frac{z f^{\prime}(z)}{f(z)}\right)<1+\frac{\alpha}{2 \sin \alpha} \quad(z \in \mathbb{U}) .
$$

Remark 1 From the inequalities (see [2])

$$
1-\frac{\pi}{4} \leq 1+\frac{\alpha-\pi}{2 \sin \alpha}<\frac{1}{2} \quad \text { and } \quad 1+\frac{\alpha}{2 \sin \alpha} \geq 1+\frac{\pi}{4} \quad(\pi / 2 \leq \alpha<\pi)
$$

it is clear that

$$
\mathcal{M S}(\alpha) \subset \mathcal{S}^{*} \quad(\pi / 2 \leq \alpha<\pi) \quad \text { and } \quad \mathcal{M S}(\pi / 2) \subset \mathcal{S}(1-\pi / 4,1+\pi / 4)
$$

where the class $\mathcal{S}(\beta, \gamma), 0 \leq \beta<1<\gamma$, was considered recently by Kwon et al. [4]. 
This paper is organized as follows. In Sect. 2, we recall certain preliminary lemmas, which are useful in the study of the mentioned classes of functions. In Sect. 3, we consider some basic properties of the class $\mathcal{M S}(\alpha)$, such as integral representation, property of convolution, sufficient condition, and coefficient inequalities. In Sect. 4, we consider radius problems and inclusion relations for certain classes of strongly starlike functions, parabolic starlike functions, and $\mathcal{S} \mathcal{L} \subset \mathcal{S}^{*}$, which are closely related to the class $\mathcal{M S}(\alpha)$, and the derivations are similar to those used earlier by Sun et al. [13] and Kwon et al. [4]. Our results are essential improvements of the corresponding results obtained by Kargar et al. [2].

\section{Preliminaries}

Recently, Kargar et al. [2] introduced the analytic function $F_{\alpha}$ and the vertical strip $\Omega_{\alpha}$ defined as follows:

$$
F_{\alpha}(z):=\frac{1}{2 i \sin \alpha} \log \left(\frac{1+e^{i \alpha} z}{1+e^{-i \alpha} z}\right) \quad(z \in \mathbb{U})
$$

and

$$
\Omega_{\alpha}:=\left\{\omega \in \mathbb{C}: \frac{\alpha-\pi}{2 \sin \alpha}<\Re(\omega)<\frac{\alpha}{2 \sin \alpha}\right\},
$$

where $\pi / 2 \leq \alpha<\pi$. The function $F_{\alpha}$ defined by (2.1) is convex and univalent in $\mathbb{U}$. In addition, $F_{\alpha}$ maps $\mathbb{U}$ onto $\Omega_{\alpha}$ or onto the convex hull of three points (one of which may be at infinity) on the boundary of $\Omega_{\alpha}$. In other words, the image of $\mathbb{U}$ may be a vertical strip for $\pi / 2 \leq \alpha<\pi$. In other cases, the image can be, for example, a half strip, a quadrilateral, or a triangle (see [1]).

Note that the function $F_{\alpha}$ can be written in the form

$$
F_{\alpha}(z)=z+\sum_{n=2}^{\infty} B_{n}(\alpha) z^{n} \quad(\pi / 2 \leq \alpha<\pi ; z \in \mathbb{U})
$$

where

$$
B_{n}(\alpha)=(-1)^{(n-1)} \frac{\sin n \alpha}{n \sin \alpha} \quad(\pi / 2 \leq \alpha<\pi ; n \in \mathbb{N}) .
$$

In the recent years, there has been significant interesting results about the class of normalized analytic functions $f \in \mathcal{A}$ that map $\mathbb{U}$ onto vertical strip; see, for example, [3-5, 9, $10,13,14]$.

To prove the main results, we need the following lemmas.

Lemma 1 (see [2]) Let $f \in \mathcal{A}$. Then $f \in \mathcal{M S}(\alpha)(\pi / 2 \leq \alpha<\pi)$ if and only if

$$
\left(\frac{z f^{\prime}(z)}{f(z)}-1\right) \prec F_{\alpha}(z)=\frac{1}{2 i \sin \alpha} \log \left(\frac{1+e^{i \alpha} z}{1+e^{-i \alpha} z}\right) \quad(z \in \mathbb{U}) .
$$

Lemma 2 (see [6]) Let $h$ be analytic and convex univalent in $\mathbb{U}$, and let $\beta, \gamma \in \mathbb{R}$ with $\Re(\beta h(z)+\gamma) \geq 0$. If $q$ is analytic in $\mathbb{U}$ with $q(0)=h(0)$, then

$$
q(z)+\frac{z q^{\prime}(z)}{\beta q(z)+\gamma} \prec h(z) \quad \Longrightarrow \quad q(z) \prec h(z) \quad(z \in \mathbb{U}) .
$$


Lemma 3 (see [7]) Let the function $r(z)$ given by

$$
r(z)=\sum_{n=1}^{\infty} C_{n} z^{n}
$$

be analytic and univalent in $\mathbb{U}$, and suppose that $r(z)$ maps $\mathbb{U}$ onto a convex domain. Suppose that the function

$$
q(z)=\sum_{n=1}^{\infty} A_{n} z^{n}
$$

is analytic in $\mathbb{U}$ and satisfies the following subordination relation:

$$
q(z) \prec r(z) \quad(z \in \mathbb{U}) .
$$

Then

$$
\left|A_{n}\right| \leq\left|C_{1}\right| \quad(n \in \mathbb{N})
$$

\section{Properties of the class $\mathcal{M S}(\alpha)$}

In this section, we study the properties of the class $\mathcal{M S}(\alpha)$. We begin by giving an integral representation for this class.

Theorem 1 A function $f \in \mathcal{M S}(\alpha)(\pi / 2 \leq \alpha<\pi)$ if and only if

$$
f(z)=z \cdot \exp \left[\frac{1}{2 i \sin \alpha} \int_{0}^{z} \frac{1}{t} \log \left(\frac{1+e^{i \alpha} w(t)}{1+e^{-i \alpha} w(t)}\right) d t\right] \quad(z \in \mathbb{U}),
$$

where $w(z)$ is a Schwarz function.

Proof For $f \in \mathcal{M S}(\alpha)$, we know from Lemma 1 that (2.4) holds. It follows that

$$
\frac{z f^{\prime}(z)}{f(z)}-1=\frac{1}{2 i \sin \alpha} \log \left(\frac{1+e^{i \alpha} w(z)}{1+e^{-i \alpha} w(z)}\right) \quad(z \in \mathbb{U})
$$

where the Schwarz function $w(z)$ is analytic in $\mathbb{U}$ with $w(0)=0$ and $|w(z)|<1(z \in \mathbb{U})$. We next see from (3.2) that

$$
\frac{f^{\prime}(z)}{f(z)}-\frac{1}{z}=\frac{1}{2 i z \sin \alpha} \log \left(\frac{1+e^{i \alpha} w(z)}{1+e^{-i \alpha} w(z)}\right)
$$

which, upon integration, yields

$$
\log \left(\frac{f(z)}{z}\right)=\frac{1}{2 i \sin \alpha} \int_{0}^{z} \frac{1}{t} \log \left(\frac{1+e^{i \alpha} w(t)}{1+e^{-i \alpha} w(t)}\right) d t
$$

Assertion (3.1) of Theorem 1 now follows from (3.3). 
Example 1 Let $w(z)=z$ in Theorem 1 . Then the function $f_{\alpha} \in \mathcal{M S}(\alpha)(\pi / 2 \leq \alpha<\pi)$ is given by

$$
f_{\alpha}(z)=z \cdot \exp \left[\frac{1}{2 i \sin \alpha} \int_{0}^{z} \frac{1}{t} \log \left(\frac{1+e^{i \alpha} t}{1+e^{-i \alpha} t}\right) d t\right] \quad(z \in \mathbb{U}) .
$$

Next, we give the following property concerning convolutions for the function class $\mathcal{M S}(\alpha)$

Theorem 2 A function $f \in \mathcal{M S}(\alpha)(\pi / 2 \leq \alpha<\pi)$ if and only if

$$
f(z) *\left\{\frac{z^{2}}{(1-z)^{2}}-\frac{z}{1-z} \cdot \frac{1}{2 i \sin \alpha} \log \left(\frac{1+e^{i(\theta+\alpha)}}{1+e^{i(\theta-\alpha)}}\right)\right\} \neq 0 \quad(z \in \mathbb{U})
$$

where $*$ denotes the Hadamard product, $0<\theta<2 \pi$, and $\theta-\alpha \neq \pi$.

Proof Assume that $f \in \mathcal{M S}(\alpha)$. Then, by Lemma 1, we observe that (2.4) holds. This implies that

$$
\frac{z f^{\prime}(z)}{f(z)} \neq 1+\frac{1}{2 i \sin \alpha} \log \left(\frac{1+e^{i \alpha} e^{i \theta}}{1+e^{-i \alpha} e^{i \theta}}\right) \quad(0<\theta<2 \pi, \theta-\alpha \neq \pi ; z \in \mathbb{U}) .
$$

Condition (3.5) can now be written as follows:

$$
z f^{\prime}(z)-\left[1+\frac{1}{2 i \sin \alpha} \log \left(\frac{1+e^{i(\theta+\alpha)}}{1+e^{i(\theta-\alpha)}}\right)\right] f(z) \neq 0 \quad(0<\theta<2 \pi, \theta-\alpha \neq \pi ; z \in \mathbb{U}) .
$$

Note that

$$
f(z)=f(z) *\left(\frac{z}{1-z}\right) \quad \text { and } \quad z f^{\prime}(z)=f(z) *\left(\frac{z}{(1-z)^{2}}\right) .
$$

Thus by (3.6) and (3.7) we obtain assertion (3.4) of Theorem 2 .

We now derive a sufficient condition involving subordination for the functions to be in the class $\mathcal{M S}(\alpha)$.

Theorem 3 Let $f \in \mathcal{A}$ satisfy the subordination

$$
\left(1+\frac{z f^{\prime \prime}(z)}{f^{\prime}(z)}\right) \prec 1+F_{\alpha}(z) \quad(z \in \mathbb{U})
$$

Then

$$
\frac{z f^{\prime}(z)}{f(z)} \prec 1+F_{\alpha}(z) \quad(z \in \mathbb{U})
$$

that is, $f \in \mathcal{M S}(\alpha)$, where $F_{\alpha}$ is given by (2.1).

Proof Consider the function $p(z)$ such that

$$
p(z)+1=\frac{z f^{\prime}(z)}{f(z)} \quad(z \in \mathbb{U}) .
$$


Then

$$
\log (p(z)+1)+\log \frac{f(z)}{z}=\log f^{\prime}(z) .
$$

We have

$$
\frac{p^{\prime}(z)}{p(z)+1}+\frac{f^{\prime}(z)}{f(z)}-\frac{1}{z}=\frac{f^{\prime \prime}(z)}{f^{\prime}(z)}
$$

or

$$
\frac{z p^{\prime}(z)}{p(z)+1}+\frac{z f^{\prime}(z)}{f(z)}-1=\frac{z f^{\prime \prime}(z)}{f^{\prime}(z)}
$$

From (3.8) and (3.10) we have

$$
\frac{z p^{\prime}(z)}{p(z)+1}+p(z)=\frac{z f^{\prime \prime}(z)}{f^{\prime}(z)} \prec F_{\alpha}(z)
$$

Note that

$$
p(0)=0=F_{\alpha}(0) \quad \text { and } \quad \Re\left(1+F_{\alpha}(z)\right)>0 \quad(\pi / 2 \leq \alpha<\pi ; z \in \mathbb{U}) \text {. }
$$

Moreover, by (3.11) and (3.12) in Lemma 2 we have

$$
p(z) \prec F_{\alpha}(z),
$$

or by (3.10) we have

$$
\frac{z f^{\prime}(z)}{f(z)} \prec 1+F_{\alpha}(z) \quad(z \in \mathbb{U})
$$

Therefore by Lemma 1 we obtain that $f \in \mathcal{M S}(\alpha)$.

Remark 2 It is well known that $\mathcal{K} \subset \mathcal{S}^{*}(1 / 2)$. In view of (1.3) and Theorem 3, we can obtain $\mathcal{K}(\Phi(\alpha)) \subset \mathcal{S}^{*}(\Phi(\alpha))$ for $\pi / 2 \leq \alpha<\pi$, where $\Phi(\alpha)=1+(\alpha-\pi) /(2 \sin \alpha)$.

Now, we present bounds for the coefficients of functions of the class $\mathcal{M S}(\alpha)$. The basic method of proof is similar to that used in [12, Thm. 3.1].

Theorem $4 \operatorname{Let} f(z)=z+\sum_{n=2}^{\infty} a_{n} z^{n} \in \mathcal{M S}(\alpha)$. Then

$$
\left|a_{n}\right| \leq 1 \quad(n \in \mathbb{N}) .
$$

Proof For given $\alpha(\pi / 2 \leq \alpha<\pi)$, we define the functions $q(z)$ and $p(z)$ by

$$
q(z)=\frac{z f^{\prime}(z)}{f(z)} \quad(z \in \mathbb{U})
$$


and

$$
p_{\alpha}(z)=1+\frac{1}{2 i \sin \alpha} \log \left(\frac{1+e^{i \alpha} z}{1+e^{-i \alpha} z}\right) \quad(z \in \mathbb{U})
$$

Then the subordination (2.4) can be written as follows:

$$
q(z) \prec p_{\alpha}(z) \quad(z \in \mathbb{U})
$$

Note that the function $p(z)$ defined by (3.14) is convex in $\mathbb{U}$ and has the form

$$
p_{\alpha}(z)=1+\sum_{n=1}^{\infty} B_{n}(\alpha) z^{n} \quad(z \in \mathbb{U})
$$

where $B_{n}(\alpha)$ is given by (2.3). If we let

$$
q(z)=1+\sum_{n=1}^{\infty} A_{n} z^{n} \quad(z \in \mathbb{U})
$$

then by Lemma 3 we see that the subordination (3.15) implies that

$$
\left|A_{n}\right| \leq\left|B_{1}\right|=1 \quad(n \in \mathbb{N}) .
$$

Now (3.13) implies that

$$
z f^{\prime}(z)=f(z) q(z) \quad(z \in \mathbb{U})
$$

Then by equating the coefficients of $z^{n}$ on both sides we get

$$
a_{n}=\frac{1}{n-1}\left(A_{n-1}+a_{2} A_{n-2}+a_{3} A_{n-3}+\cdots+a_{n-1} A_{1}\right) \quad(n \in \mathbb{N} \backslash\{1\}) .
$$

A simple calculation combined with inequality (3.16) yields $\left|a_{2}\right|=\left|A_{1}\right| \leq 1$ and

$$
\begin{aligned}
\left|a_{n}\right| & =\frac{1}{n-1}\left|A_{n-1}+a_{2} A_{n-2}+a_{3} A_{n-3}+\cdots+a_{n-1} A_{1}\right| \\
& \leq \frac{1}{n-1}\left(\left|A_{n-1}\right|+\left|a_{2}\right| \cdot\left|A_{n-2}\right|+\left|a_{3}\right| \cdot\left|A_{n-3}\right|+\cdots+\left|a_{n-1}\right| \cdot\left|A_{1}\right|\right) \\
& \leq \frac{\left|B_{1}\right|}{n-1}\left(1+\sum_{k=2}^{n-1}\left|a_{k}\right|\right)=\frac{1}{n-1}\left(1+\sum_{k=2}^{n-1}\left|a_{k}\right|\right) \quad(n \in \mathbb{N} \backslash\{1,2\}) .
\end{aligned}
$$

To prove Theorem 4, we need to show that

$$
\left|a_{n}\right| \leq \frac{1}{n-1}\left(1+\sum_{k=2}^{n-1}\left|a_{k}\right|\right) \leq 1 \quad(n \in \mathbb{N} \backslash\{1,2\})
$$

We prove (3.17) by induction. For $n=3$, we have

$$
\left|a_{3}\right| \leq \frac{1}{2}\left(1+\left|a_{2}\right|\right) \leq \frac{1}{2}\left(1+\left|A_{1}\right|\right) \leq 1 .
$$


Then suppose that inequality (3.17) is true for $3 \leq n \leq m$. We prove the statement for $n=m+1$. Straightforward calculations yield

$$
\left|a_{m+1}\right| \leq \frac{1}{m}\left(1+\sum_{k=2}^{m}\left|a_{k}\right|\right) \leq \frac{1}{m}[1+(m-1)]=1,
$$

which implies that inequality (3.17) is true for $n=m+1$.

\section{Radius problems and inclusion relations}

In this section, we first give results on the radius problem involving the function class $\mathcal{M S}(\alpha)$. As an application, we obtain inclusion relations for the class $\mathcal{M S}(\alpha)$ and the other well-known function classes. The basic method of the proof in the following theorem is similar to that used in [13, Thm. 5] (see also [4, Thm. 3.1]).

Theorem 5 Let $f \in \mathcal{M S}(\alpha)$. Then, for each $z(|z|=r<1)$,

$$
1+\frac{1}{2 \sin \alpha}\left[M_{1}(r, \alpha)-M_{2}(r, \alpha)\right] \leq \Re\left(\frac{z f^{\prime}(z)}{f(z)}\right) \leq 1+\frac{1}{2 \sin \alpha}\left[M_{1}(r, \alpha)+M_{2}(r, \alpha)\right]
$$

and

$$
\left|\Im\left(\frac{z f^{\prime}(z)}{f(z)}\right)\right| \leq \frac{1}{2 \sin \alpha} \log [N(r, \alpha)],
$$

where

$$
\begin{aligned}
& M_{1}(r, \alpha)=\arcsin \left(\frac{-r^{2} \sin 2 \alpha}{\sqrt{1-2 r^{2} \cos 2 \alpha+r^{4}}}\right), \\
& M_{2}(r, \alpha)=\arcsin \left(\frac{2 r \sin \alpha}{\sqrt{1-2 r^{2} \cos 2 \alpha+r^{4}}}\right), \\
& N(r, \alpha)=\frac{\sqrt{1-2 r^{2} \cos 2 \alpha+r^{4}}+2 r \sin \alpha}{1-r^{2}} .
\end{aligned}
$$

Proof Suppose that $f \in \mathcal{M S}(\alpha)$. Then by Lemma 1 assertion (2.4) holds. Thus by the definition of subordination there exists a Schwarz function $w(z)$ such that

$$
\frac{z f^{\prime}(z)}{f(z)}=1+\frac{1}{2 i \sin \alpha} \log \left(\frac{1+e^{i \alpha} w(z)}{1+e^{-i \alpha} w(z)}\right) \quad(z \in \mathbb{U})
$$

We put

$$
Q(z)=\frac{1+e^{i \alpha} w(z)}{1+e^{-i \alpha} w(z)} \quad(z \in \mathbb{U}),
$$

which readily yields

$$
Q(z)-1=\left[e^{i \alpha}-e^{-i \alpha} Q(z)\right] w(z) .
$$

For $|z|=r<1$, using the Schwarz lemma,

$$
|w(z)| \leq|z| \quad(z \in \mathbb{U})
$$


we find that

$$
|Q(z)-1| \leq\left|e^{i \alpha}-e^{-i \alpha} Q(z)\right| r \quad(|z|=r<1) .
$$

If we set $Q(z)=u+i v$, then upon squaring both sides of (4.6) we get

$$
\left(u-\frac{1-r^{2} \cos 2 \alpha}{1-r^{2}}\right)^{2}+\left(v+\frac{r^{2} \sin 2 \alpha}{1-r^{2}}\right)^{2} \leq\left(\frac{2 r \sin \alpha}{1-r^{2}}\right)^{2} .
$$

Thus $Q(z)$ maps the disk

$$
\overline{\mathbb{U}_{r}}=\{z: z \in \mathbb{C} \text { and }|z| \leq r<1\}
$$

onto the disk with center $C$ and radius $R$ given by

$$
C:=\left(\frac{1-r^{2} \cos 2 \alpha}{1-r^{2}},-\frac{r^{2} \sin 2 \alpha}{1-r^{2}}\right) \text { and } R:=\frac{2 r \sin \alpha}{1-r^{2}}
$$

We observe that

$$
\begin{aligned}
& X_{C}:=\frac{1-r^{2} \cos 2 \alpha}{1-r^{2}}>0, \quad Y_{C}:=-\frac{r^{2} \sin 2 \alpha}{1-r^{2}}>0, \\
& R=\frac{2 r \sin \alpha}{1-r^{2}}>0 \quad(\pi / 2 \leq \alpha<\pi),
\end{aligned}
$$

and

$$
|\overrightarrow{O C}|^{2}-R^{2}=1>0, \quad Y_{C}-R=\frac{2 r \sin \alpha(1-\cos \alpha)}{1-r^{2}}<0 \quad(\pi / 2 \leq \alpha<\pi) .
$$

Hence the origin $O$ lies outside of the disk (4.8), and the disk (4.8) lies in the first and forth quadrants of the $u v$-plane.

We can obtain upper and lower bounds of $|Q(z)|$ :

$$
|Q(z)| \leq|\overrightarrow{O C}|+R=\frac{\sqrt{1-2 r^{2} \cos 2 \alpha+r^{4}}+2 r \sin \alpha}{1-r^{2}}=: N(r, \alpha)
$$

and

$$
|Q(z)| \geq|\overrightarrow{O C}|-R=\frac{\sqrt{1-2 r^{2} \cos 2 \alpha+r^{4}}-2 r \sin \alpha}{1-r^{2}}=\frac{1}{N(r, \alpha)},
$$

where $N(r, \alpha)>1$ is already given by (4.5).

Furthermore, a simple geometric observation shows that (4.7) implies

$$
M_{1}(r, \alpha)-M_{2}(r, \alpha) \leq \arg (Q(z)) \leq M_{1}(r, \alpha)+M_{2}(r, \alpha),
$$

where $M_{1}(r, \alpha)$ and $M_{2}(r, \alpha)$ are given by (4.3) and (4.4), respectively. 
For $|z|=r<1$, we have

$$
\begin{aligned}
\frac{z f^{\prime}(z)}{f(z)} & =1+\frac{1}{2 i \sin \alpha} \log (Q(z)) \\
& =1+\frac{1}{2 i \sin \alpha}[\log |Q(z)|+i \arg (Q(z))] \\
& =1+\frac{1}{2 \sin \alpha} \arg (Q(z))-\frac{i}{2 \sin \alpha} \log |Q(z)|
\end{aligned}
$$

Thus by (4.9)-(4.12) we easily get assertions (4.1) and (4.2) of Theorem 5.

The following identities are used in the proofs of our main results:

$$
\lim _{r \rightarrow 0+} M_{1}(r, \alpha)=\lim _{r \rightarrow 0+} M_{2}(r, \alpha)=0, \quad \lim _{r \rightarrow 0+} N(r, \alpha)=1,
$$

and

$$
\lim _{r \rightarrow 1-} M_{1}(r, \alpha)=\frac{3 \pi}{2}-\alpha, \quad \lim _{r \rightarrow 1-} M_{2}(r, \alpha)=\frac{\pi}{2}, \quad \lim _{r \rightarrow 1-} N(r, \alpha)=+\infty,
$$

where $M_{1}(r, \alpha), M_{2}(r, \alpha)$, and $N(r, \alpha)$ are given by (4.3), (4.4), and (4.5), respectively.

Using Theorem 5, we derive the following inclusion relations for the class $\mathcal{M S}(\alpha)$.

\section{Theorem 6 Let}

$$
\frac{\pi}{2} \leq \alpha<\pi \quad \text { and } \quad 0 \leq \gamma<1
$$

Then

$$
\mathcal{M S}(\alpha) \subset \mathcal{S S}(\gamma) \quad\left(|z| \leq r_{1}\right)
$$

where $r_{1} \in(0,1)$ is the least positive root of the equation

$$
\arctan \left(\frac{\log [N(r, \alpha)]}{2 \sin \alpha+M_{1}(r, \alpha)-M_{2}(r, \alpha)}\right)-\frac{\pi}{2} \gamma=0 \quad(0 \leq r<1),
$$

where $M_{1}(r, \alpha), M_{2}(r, \alpha)$, and $N(r, \alpha)$ are given by (4.3), (4.4), and (4.5), respectively.

Proof We first note that

$$
1+\frac{1}{2 \sin \alpha}\left[M_{1}(r, \alpha)-M_{2}(r, \alpha)\right]>0 \quad(\pi / 2 \leq \alpha<\pi ; 0 \leq r<1) .
$$

Hence by Theorem 5 , for $f \in \mathcal{M S}(\alpha)$, we have

$$
\left|\arg \left(\frac{z f^{\prime}(z)}{f(z)}\right)\right| \leq \arctan \left(\frac{\frac{1}{2 \sin \alpha} \log [N(r, \alpha)]}{1+\frac{1}{2 \sin \alpha}\left[M_{1}(r, \alpha)-M_{2}(r, \alpha)\right]}\right) .
$$

Thus, for the function $f \in \mathcal{S S}(\gamma)$, it suffices to prove the inequality

$$
\arctan \left(\frac{\log [N(r, \alpha)]}{2 \sin \alpha+M_{1}(r, \alpha)-M_{2}(r, \alpha)}\right)-\frac{\pi}{2} \gamma<0 .
$$


We now define the continuous function

$$
G(r)=\arctan \left(\frac{\log [N(r, \alpha)]}{2 \sin \alpha+M_{1}(r, \alpha)-M_{2}(r, \alpha)}\right)-\frac{\pi}{2} \gamma \quad(0 \leq r<1) .
$$

In view of (4.13) and (4.14), we can show that

$$
G(0)=-\frac{\pi}{2} \gamma<0 \quad \text { and } \quad \lim _{r \rightarrow 1-} G(r)=\frac{\pi}{2}-\frac{\pi}{2} \gamma>0 .
$$

Thus, the equation $G(r)=0$ has a solution in $(0,1)$. Let $r_{1} \in(0,1)$ be the least positive root of $G(r)=0$. Then $G(r)<0$ for all $r<r_{1}$. Hence $f$ is a strongly starlike function of order $\gamma$ for $z\left(|z| \leq r_{1}\right)$.

Theorem 7 Let $\pi / 2 \leq \alpha<\pi$. Then

$$
\mathcal{M S}(\alpha) \subset \mathcal{P S} \quad\left(|z| \leq r_{2}\right)
$$

where $r_{2} \in(0,1)$ is the least positive root of the equation

$$
\frac{1}{4 \sin ^{2} \alpha}\{\log [N(r, \alpha)]\}^{2}-\frac{1}{\sin \alpha}\left[M_{1}(r, \alpha)-M_{2}(r, \alpha)\right]-1=0 \quad(0 \leq r<1),
$$

where $M_{1}(r, \alpha), M_{2}(r, \alpha)$, and $N(r, \alpha)$ are given by (4.3), (4.4), and (4.5), respectively.

Proof Note that $f \in \mathcal{P S}$ if and only if the function $z f^{\prime}(z) / f(z)$ is in the parabolic region given by

$$
\Lambda=\left\{(u, v): v^{2}<2 u-1\right\} .
$$

Thus by combining (4.1) and (4.2), for the function $f \in \mathcal{P S}$ in $\mathbb{U}$, it suffices to show that

$$
\left(1+\frac{1}{2 \sin \alpha}\left[M_{1}(r, \alpha)-M_{2}(r, \alpha)\right], \frac{1}{2 \sin \alpha} \log [N(r, \alpha)]\right) \in \Lambda
$$

that is,

$$
\frac{1}{4 \sin ^{2} \alpha}\{\log [N(r, \alpha)]\}^{2}-\frac{1}{\sin \alpha}\left[M_{1}(r, \alpha)-M_{2}(r, \alpha)\right]-1<0 .
$$

We now define the continuous function

$$
H(r):=\frac{1}{4 \sin ^{2} \alpha}\{\log [N(r, \alpha)]\}^{2}-\frac{1}{\sin \alpha}\left[M_{1}(r, \alpha)-M_{2}(r, \alpha)\right]-1 \quad(0 \leq r<1) .
$$

In view of (4.13) and (4.14), we have

$$
H(0)=-1<0 \quad \text { and } \quad \lim _{r \rightarrow 1-} H(r)=+\infty .
$$

Hence the equation $H(r)=0$ has a solution in $(0,1)$. Let $r_{2} \in(0,1)$ be the least positive root of $H(r)=0$. Then $H(r)<0$ for all $r<r_{2}$. Therefore we have $f \in \mathcal{P S}$ for all $z\left(|z| \leq r_{2}\right)$. 
Theorem 8 Let $\pi / 2 \leq \alpha<\pi$. Then

$$
\mathcal{M S}(\alpha) \subset \mathcal{S L} \quad\left(|z| \leq r_{0}\right)
$$

where $r_{0}:=\min \left\{r_{3}, r_{4}\right\}$, and $r_{3}, r_{4} \in(0,1)$ are the least positive root of the equations

$$
\begin{aligned}
& \left\{\left(1+\frac{1}{2 \sin \alpha}\left[M_{1}(r, \alpha)+M_{2}(r, \alpha)\right]\right)^{2}+\frac{1}{4 \sin ^{2} \alpha}(\log [N(r, \alpha)])^{2}\right\}^{2} \\
& \quad=2\left(1+\frac{1}{2 \sin \alpha}\left[M_{1}(r, \alpha)+M_{2}(r, \alpha)\right]\right)^{2}-\frac{1}{2 \sin ^{2} \alpha}(\log [N(r, \alpha)])^{2}
\end{aligned}
$$

and

$$
\begin{aligned}
& \left\{\left(1+\frac{1}{2 \sin \alpha}\left[M_{1}(r, \alpha)-M_{2}(r, \alpha)\right]\right]^{2}+\frac{1}{4 \sin ^{2} \alpha}(\log [N(r, \alpha)])^{2}\right\}^{2} \\
& \quad=2\left(1+\frac{1}{2 \sin \alpha}\left[M_{1}(r, \alpha)-M_{2}(r, \alpha)\right]\right)^{2}-\frac{1}{2 \sin ^{2} \alpha}(\log [N(r, \alpha)])^{2},
\end{aligned}
$$

respectively, where $M_{1}(r, \alpha), M_{2}(r, \alpha)$, and $N(r, \alpha)$ are given by (4.3), (4.4), and (4.5), respectively.

Proof Note that $f \in \mathcal{S} \mathcal{L}$ if and only if the function $z f^{\prime}(z) / f(z)$ is in the bounded region given by

$$
\Xi=\left\{(u, v):\left(u^{2}+v^{2}\right)^{2}<2\left(u^{2}-v^{2}\right)\right\}
$$

Thus by combining (4.1) and (4.2), for the function $f \in \mathcal{S} \mathcal{L}$ in $\mathbb{U}$, it suffices to show that

$$
\left(1+\frac{1}{2 \sin \alpha}\left[M_{1}(r, \alpha) \pm M_{2}(r, \alpha)\right], \frac{1}{2 \sin \alpha} \log [N(r, \alpha)]\right) \in \Xi
$$

that is,

$$
\begin{aligned}
& \left\{\left(1+\frac{1}{2 \sin \alpha}\left[M_{1}(r, \alpha)+M_{2}(r, \alpha)\right]\right)^{2}+\frac{1}{4 \sin ^{2} \alpha}(\log [N(r, \alpha)])^{2}\right\}^{2} \\
& \quad<2\left(1+\frac{1}{2 \sin \alpha}\left[M_{1}(r, \alpha)+M_{2}(r, \alpha)\right]\right)^{2}-\frac{1}{2 \sin ^{2} \alpha}(\log [N(r, \alpha)])^{2}
\end{aligned}
$$

and

$$
\begin{aligned}
& \left\{\left(1+\frac{1}{2 \sin \alpha}\left[M_{1}(r, \alpha)-M_{2}(r, \alpha)\right]\right)^{2}+\frac{1}{4 \sin ^{2} \alpha}(\log [N(r, \alpha)])^{2}\right\}^{2} \\
& \quad<2\left(1+\frac{1}{2 \sin \alpha}\left[M_{1}(r, \alpha)-M_{2}(r, \alpha)\right]\right)^{2}-\frac{1}{2 \sin ^{2} \alpha}(\log [N(r, \alpha)])^{2}
\end{aligned}
$$


Table 1 The radii of inclusion relations

\begin{tabular}{lll}
\hline Inclusion relations & Radii in this paper & Radii in [2] \\
\hline $\mathcal{M S}(\pi / 2) \subset \mathcal{S S}(1 / 2)\left(|z| \leq r_{1}\right)$ & $r_{1} \approx 0.493918$ & $r_{1} \approx 0.260446$ \\
$\mathcal{M S}(\pi / 2) \subset \mathcal{P S}\left(|z| \leq r_{2}\right)$ & $r_{2} \approx 0.421547$ & $r_{2} \approx 0.246969$ \\
$\mathcal{M S}(\pi / 2) \subset \mathcal{S} \mathcal{L}\left(|z| \leq r_{0}\right)$ & $r_{0} \approx 0.304506$ & $r_{0} \approx 0.200667$ \\
\hline
\end{tabular}

respectively. We define the continuous function

$$
\begin{aligned}
P(r):= & \left\{\left(1+\frac{1}{2 \sin \alpha}\left[M_{1}(r, \alpha)+M_{2}(r, \alpha)\right]\right)^{2}+\frac{1}{4 \sin ^{2} \alpha}(\log [N(r, \alpha)])^{2}\right\}^{2} \\
& -2\left(1+\frac{1}{2 \sin \alpha}\left[M_{1}(r, \alpha)+M_{2}(r, \alpha)\right]\right)^{2}+\frac{1}{2 \sin ^{2} \alpha}(\log [N(r, \alpha)])^{2} \quad(0 \leq r<1) .
\end{aligned}
$$

In view of (4.13) and (4.14), we have

$$
P(0)=-1<0 \quad \text { and } \quad \lim _{r \rightarrow 1-} P(r)=+\infty
$$

Hence the equation $P(r)=0$ has a solution in $(0,1)$. Let $r_{3} \in(0,1)$ be the least positive root of $H(r)=0$. Then $P(r)<0$ for all $r<r_{3}$. Using the same approach as before, we can find $r_{4} \in(0,1)$ to be the least positive root of equation (4.16), and inequality (4.18) holds for all $r<r_{4}$. So if we take $r_{0}:=\min \left\{r_{3}, r_{4}\right\}$, then we have $f \in \mathcal{S} \mathcal{L}$ for all $z\left(|z| \leq r_{0}\right)$.

Remark 3 Putting $\alpha=\pi / 2$ in Theorems $6-8$, we obtain the radii of inclusion relations between several known classes and the class $\mathcal{M S}(\alpha)$. Furthermore, the results are compared with the corresponding results in [2] (see Table 1).

\section{Funding}

The present investigation was supported by the Natural Science Foundation of Hunan Province under Grant no. 2016JJ2036 of the People's Republic of China.

\section{Competing interests}

The authors declare that they have no competing interests.

\section{Authors' contributions}

All authors read and approved the final manuscript.

\section{Author details}

${ }^{1}$ School of Science, Hunan Institute of Engineering, Xiangtan, People's Republic of China. ${ }^{2}$ School of Mathematics and Computing Science, Hunan First Normal University, Changsha, People's Republic of China. ${ }^{3}$ Guangdong Technion-Israel Institute of Technology, Shantou, People's Republic of China. ${ }^{4}$ Faculty of Mathematics and Natural Sciences, University of Rzeszów, Rzeszów, Poland.

\section{Publisher's Note}

Springer Nature remains neutral with regard to jurisdictional claims in published maps and institutional affiliations.

Received: 17 March 2018 Accepted: 29 January 2019 Published online: 06 February 2019

\section{References}

1. Dorff, M.: Convolutions of planar harmonic convex mappings. Complex Var. Theory Appl. 45, 263-271 (2001)

2. Kargar, R., Ebadian, A., Sokół, J.: Radius problems for some subclasses of analytic functions. Complex Anal. Oper. Theory 11, 1639-1649 (2017)

3. Kuroki, K., Owa, S.: Notes on new class for certain analytic functions. RIMS Kôkyûroku Kyoto Univ. 1772, 21-25 (2011)

4. Kwon, O.S., Sim, Y.J., Cho, N.E., Srivastava, H.M.: Some radius problems related to a certain subclass of analytic functions. Acta Math. Sin. Engl. Ser. 30, 1133-1144 (2014)

5. Li, S.-H., Tang, H., Ma, N.-A., Niu, X.-M.: Coefficient estimates for the subclasses of analytic functions and bi-univalent functions associated with the strip domain. J. Math. Res. Appl. 37, 550-562 (2017) 
6. Miller, S.S., Mocanu, P.T.: Second-order differential inequalities in the complex plane. J. Math. Anal. Appl. 65, 289-305 (1978)

7. Rogosinski, W.: On the coefficients of subordinate functions. Proc. Lond. Math. Soc. (2) 48, 48-82 (1943)

8. Rønning, F.: Uniformly convex functions and a corresponding class of starlike functions. Proc. Am. Math. Soc. 118 189-196 (1993)

9. Sim, Y.J., Kwon, O.S.: On certain classes of convex functions. Int. J. Math. Math. Sci. 2013, Article ID 294378 (2013)

10. Sim, Y.J., Kwon, O.S.: Some results on analytic functions associated with vertical strip domain. Proc. Jangjeon Math. Soc. 19, 653-661 (2016)

11. Sokół, J.: On some subclass of strongly starlike functions. Demonstr. Math. 21, 81-86 (1998)

12. Sun, Y., Jiang, Y.-P., Rasila, A.: Coefficient estimates for certain subclasses of analytic and bi-univalent functions. Filomat 29, 351-360 (2015)

13. Sun, Y., Jiang, Y.P., Rasila, A., Srivastava, H.M.: Integral representations and coefficient estimates for a subclass of meromorphic starlike functions. Complex Anal. Oper. Theory 11, 1-19 (2017)

14. Wang, Z.-G., Shi, L., Jiang, Y.-P.: On harmonic K-quasiconformal mappings associated with asymmetric vertical strips. Acta Math. Sin. Engl. Ser. 31, 1970-1976 (2015)

\section{Submit your manuscript to a SpringerOpen ${ }^{\circ}$} journal and benefit from:

- Convenient online submission

- Rigorous peer review

- Open access: articles freely available online

- High visibility within the field

- Retaining the copyright to your article

Submit your next manuscript at $\boldsymbol{\nabla}$ springeropen.com 